\title{
Probability Theory Predicts That Group Survival May Be Guaranteed for Groups with More Than 10 Elements
}

\author{
Motohisa Osaka \\ Department of Basic Science, Nippon Veterinary and Life Science University, Tokyo, Japan \\ Email: osaka@nms.ac.jp
}

How to cite this paper: Osaka, M. (2017) Probability Theory Predicts That Group Survival May Be Guaranteed for Groups with More Than 10 Elements. Applied Mathematics, 8, 1745-1760.

https://doi.org/10.4236/am.2017.812125

Received: November 6, 2017

Accepted: December 2, 2017

Published: December 5, 2017

Copyright $\odot 2017$ by author and Scientific Research Publishing Inc. This work is licensed under the Creative Commons Attribution International License (CC BY 4.0).

http://creativecommons.org/licenses/by/4.0/

\begin{abstract}
The purpose of this study is to find out the critical number of elements needed for group survival. Taking a probabilistic approach, how the lifetime of a group consisting of several elements depends on the number of elements and the probability distribution of their lifetimes is investigated. Four probability distributions are examined: an exponential distribution, a uniform distribution, a parabolic distribution, and a pointed distribution composed of two parabolas. The lifetime of the group is defined as the expected value of the maximum lifetime of the elements in that group. The probability distribution of this maximum shifts to the right as the number of elements increases, and the expected value of the lifetime of each element eventually becomes less than the lower limit of this distribution. The number of elements in this case is defined as the critical number of elements needed for group survival. Hence, if the number of elements is larger than the critical number needed for group survival, the lifetime of the group is guaranteed to be longer than the expected lifetime of one element. The findings are in the following. The critical number needed for group survival is inversely proportional to the expected lifetime of one element, regardless of the probability distribution used. It decreases as $\left(\frac{2}{3}\right)^{N-1}$ for an exponential distribution, and as $\left(\frac{1}{2}\right)^{N-1}$ in case of the others,
\end{abstract} where $N$ is the number of elements of the group. Because a probability distribution defined over a finite range is assumed to be reasonable in practice, a group consisting of more than 10 elements should survive well.

\section{Keywords}

Lifetime, Neuron, Cerebral Atrophy, Hippocampus, Memory 


\section{Introduction}

Consider the following scenario: Suppose that 5 years ago, four new light bulbs were placed in the dining room of a house. Further suppose that one of them stopped working after 4 years. However, the three remaining light bulbs were sufficiently bright for daily life. Thereafter, one of these three stopped working after another year, but even two bulbs provided sufficient brightness. If only one light bulb had been installed at the beginning, the room could have become dark after only 4 years. Another example is an electrical circuit that consists of 10 equivalent elements. If any one of the elements stops working, one of the others works instead and the circuit will work until the last element stops working. The life (or service) times of these elements are different from each other and follow a probability distribution. If the size of the circuit is large, in other words, the number of elements is large, it is presumed that at least one of the elements is likely to have a lifetime that is substantially longer than the average lifetime. Hence, it is generally expected that the lifetime of a group will be longer when its size is larger. The present study examines how the lifetime of a group depends on the number of its elements and the probability distribution of the elements' lifetimes. The lifetime of a group is defined as the expected value of the maximum of the lifetimes of the elements in the group.

One example in the medical field that demonstrates the advantages of a group of elements over a single element is cardiac pacemaker cell synchronization [1]. Each pacemaker cell of the sinoatrial node (a group of pacemaker cells in the wall of the right atrium of the heart) is loosely coupled with its neighboring pacemaker cells so that those cells synchronize. The electrical impulse current of a single pacemaker cell is not sufficient to travel through the impulse-conducting system, so the ventricles of the heart do not contract. In contrast, synchronized pacemaker cells generate an impulse with a sufficiently high current to travel through the system so that the ventricles contract and pump out blood normally. Hence, the failure of this synchronization is presumed to cause sinoatrial arrest [2]. Sinoatrial arrest is a part of sick sinus syndrome, the symptoms of which include fainting, vertigo, and weakness. Computer simulations have shown that the pacemaker cells synchronize more easily when their number is larger. The sinus node consists of thousands of pacemaker cells [3], and the incidence of sick sinus syndrome increases with aging [4]. It has hence been suggested that the number of pacemaker cells that survive into advanced age is not sufficient for synchronization.

Some degree of cerebral atrophy occurs naturally with aging. Brain weight is maximum at approximately age 25 , and gradually decreases by $0.22 \%$ per year [5]. Cerebral atrophy is microscopically due to a loss of neurons and the connections between them. Oxidized proteins increase in older cerebral cortexes because of free radical-mediated oxidation, which occurs in the aging process. Moreover, oxidative damage to DNA also occurs in the brain [6]. Pathological atrophy causes dementia, $60 \%-70 \%$ of which is Alzheimer's disease. Even con- 
sidering the physiological atrophy that occurs with aging, by the time the cerebral cortex is 70 years old, it has lost about $15 \%$ of the mass it had at age 25 . This loss does not necessarily cause dementia [7] but indicates that the brain has lost functional spare capacity. Hence, it is speculated that neurons that stop working because of aging may be replaced by spare ones that have a longer lifetime.

Anatomically, minicolumns (23- $\mu \mathrm{m}$ wide) are the minimal local nervous circuits in the human cortex. Each of them consists of 80 neurons that are arranged longitudinally. These are arrayed repeatedly in the lateral direction. There are roughly 2.6 billion of these neurons [8] [9] [10]. Each minicolumn has an excitatory part consisting of pyramidal cells and an inhibitory part of non-pyramidal cells [11] [12]. In the monkey primary visual cortex, the excitatory part includes three subparts of 18,6 , and 10 pyramidal cells. The excitatory part is surrounded by the inhibitory part, and these neurons are connected with each other. Hence, these parts are considered to compose one functional unit. A functional column (400- $\mu \mathrm{m}$ wide) consists of hundreds of minicolumns so that it responds to similar visual patterns. This prompts the question whether fewer than hundreds of minicolumns are sufficient for functionality. The primary visual cortex belongs to the occipital lobes, and vision is retained even at advanced age. Hence, it is presumed that any impaired minicolumn is replaced functionally by other ones with longer lifetimes in the same unit.

In the above paragraphs, a few examples are described to focus on the fact that a group of elements has the advantage of functional maintenance over a single element. The present study examines how the probability distribution of the lifetime of elements in a group affects the expected value of their maximum lifetime, in other words, the overall life time of the group.

\section{Model}

Nahin published an interesting book titled Will You Be Alive 10 Years from Now? [13]. In Chapter 19 ("When Will the Last One Fail?"), an exponential distribution was employed as the probability distribution of the lifetimes of elements in a group. The exponential distribution is the probability distribution that describes the time between events in a process in which events occur independently at a constant average rate. Hence, if the elements of a group fail independently at a constant average rate, the lifetime of each element follows an exponential distribution. Hence, an exponential distribution is first used to examine how the probability distribution of element lifetimes affects the expected value of their maximum lifetime. The range of the random variable lifetime $T$ is between 0 and positive infinity; however, positive infinity is not a realistic value. Moreover, an exponential distribution has the specific property of being memoryless. It is expressed using conditional probability in the following form:

$$
\operatorname{Prob}(T>a+b \mid T>a)=\operatorname{Prob}(T>b),
$$

where $T$ is a random variable and $a$ and $b$ are any non-negative values. The Formula (1) means that the probability an element will survive for an addition 
time interval of $T>b$ is independent of how long it has already been alive. However, the value of the probability density is maximum at $T=0$ in the exponential distribution, and the lifetime of a cell is shorter as an organism ages. These facts indicate that the exponential distribution may not necessarily be applicable to biological and medical fields. Hence, this problem is also examined using the following probability distributions of $T$ defined over a finite range: a uniform distribution, parabolic distribution, and pointed distribution composed of two parabolas.

Because $t$ is time in the present study, $t \geq 0$. The distribution function $F_{M}(t)$ for the maximum $M$ of $N$ independent, identically distributed random variables $T_{p}$, which are the lifetime of the $i$-th element of the group $(1 \leq i \leq N)$, is defined as:

$$
F_{M}(t)=\operatorname{Prob}(M \leq t)=\operatorname{Prob}\left(\max _{1 \leq i \leq N}\left\{T_{i}\right\} \leq t\right) .
$$

Because the largest $T_{i} \leq t$ and the $T_{i}$ are independent,

$$
\begin{aligned}
F_{M}(t) & =\operatorname{Prob}\left(T_{1} \leq t, T_{2} \leq t, \cdots, T_{N} \leq t\right) \\
& =\operatorname{Prob}\left(T_{1} \leq t\right) \operatorname{Prob}\left(T_{2} \leq t\right) \cdots \operatorname{Prob}\left(T_{N} \leq t\right) .
\end{aligned}
$$

When the distribution function $F_{T}(t)$ for each $T_{i}$ is defined as

$$
F_{T}(t)=\operatorname{Prob}(T \leq t),
$$

then

$$
F_{M}(t)=\left[F_{T}(t)\right]^{N} .
$$

The probability density function $f_{M}(t)$ of $M$ is given in the following form:

$$
f_{M}(t)=\frac{\mathrm{d}}{\mathrm{d} t}\left[F_{T}(t)\right]^{N}=N\left[F_{T}(t)\right]^{N-1} f_{T}(t),
$$

where $f_{T}(t)$ is the probability density function of each $T_{i}$.

Because $F_{T}(E(T))<1: E(T)$, the expected value of the lifetime $T$ of each element, $f_{M}(E(T))$ decreases and eventually approaches zero as $N$ increases. When $E(T)$ becomes less than the lower limit of the probability distribution of $M$, the value of $N$ is defined as the critical number of elements needed for group survival. We consider the specific case for each of the four probability distributions below.

\subsection{Exponential Distribution}

If the lifetime of each element is denoted as a random variable $T$,

$$
\operatorname{Prob}(T>t)=\mathrm{e}^{-\lambda t}, t \geq 0,
$$

where $\lambda$ is some positive constant, then,

$$
\begin{gathered}
F_{T}(t)=\operatorname{Prob}(T \leq t)=1-\mathrm{e}^{-\lambda t}, \\
f_{T}(t)=\frac{\mathrm{d} F_{T}(t)}{\mathrm{d} t}=\lambda \mathrm{e}^{-\lambda t} .
\end{gathered}
$$

From these equations, 


$$
f_{M}(t)=N\left[F_{T}(t)\right]^{N-1} f_{T}(t)=N\left[1-\mathrm{e}^{-\lambda t}\right]^{N-1} \lambda \mathrm{e}^{-\lambda t} .
$$

The expected value of $M$ is given by

$$
\begin{aligned}
E(M) & =\int_{0}^{\infty} t f_{M}(t) \mathrm{d} t=\int_{0}^{\infty} t N\left[1-\mathrm{e}^{-\lambda t}\right]^{N-1} \lambda \mathrm{e}^{-\lambda t} \mathrm{~d} t \\
& =\lambda N \int_{0}^{\infty} t\left[1-\mathrm{e}^{-\lambda t}\right]^{N-1} \mathrm{e}^{-\lambda t} \mathrm{~d} t \\
& =\lambda N \int_{0}^{\infty} t \sum_{k=0}^{N-1}\left(\begin{array}{c}
N-1 \\
k
\end{array}\right)(-1)^{N-1-k} \mathrm{e}^{-\lambda(N-1-k) t} \mathrm{e}^{-\lambda t} \mathrm{~d} t \\
& =\lambda N \sum_{k=0}^{N-1}\left(\begin{array}{c}
N-1 \\
k
\end{array}\right)(-1)^{N-1-k} \int_{0}^{\infty} t \mathrm{e}^{-\lambda(N-k) t} \mathrm{~d} t \\
& =N \sum_{k=0}^{N-1}\left(\begin{array}{c}
N-1 \\
k
\end{array}\right)(-1)^{N-1-k} \frac{1}{\lambda(N-k)^{2}} .
\end{aligned}
$$

The expected value of the lifetime $T$ of each element is given as

$$
E(T)=\int_{0}^{\infty} t f_{T}(t) \mathrm{d} t=\int_{0}^{\infty} t \lambda \mathrm{e}^{-\lambda t} \mathrm{~d} t=\frac{1}{\lambda} .
$$

Then,

$$
\begin{gathered}
f_{M}(E(T))=N\left[1-\mathrm{e}^{-1}\right]^{N-1} \lambda \mathrm{e}^{-1} \approx \frac{\lambda N}{3}\left(\frac{2}{3}\right)^{N-1} ; \mathrm{e}^{-1} \approx 0.3679 \\
=\frac{N}{3} \frac{1}{E(T)}\left(\frac{2}{3}\right)^{N-1}
\end{gathered}
$$

\subsection{Finite-Range Probability Distributions}

\subsubsection{Uniform Distribution}

The probability density function of $T$ is given in the following form:

$$
f_{T}(t)=\left\{\begin{array}{lc}
\frac{1}{a}, & 0 \leq t \leq a \\
0, & a<t
\end{array},\right.
$$

where $a$ is some positive constant. Hence,

$$
F_{T}(t)=\left\{\begin{array}{ll}
\frac{t}{a}, & 0 \leq t \leq a \\
1, & a<t
\end{array} .\right.
$$

Because probability density function $f_{T}(t)$ is constant in the range $0 \leq t \leq a$, the lifetime of each element is quite variable. From these equations,

$$
f_{M}(t)=N\left[F_{T}(t)\right]^{N-1} f_{T}(t)=N\left[\frac{t}{a}\right]^{N-1} \frac{1}{a} .
$$

The expected value of $M$ is given by

$$
E(M)=\int_{0}^{a} t f_{M}(t) \mathrm{d} t=\int_{0}^{a} t N\left[\frac{t}{a}\right]^{N-1} \frac{1}{a} \mathrm{~d} t=\frac{N}{N+1} a .
$$

The expected value of the lifetime $T$ of each element is given as

$$
E(T)=\int_{0}^{a} t f_{T}(t) \mathrm{d} t=\int_{0}^{a} t \frac{1}{a} \mathrm{~d} t=\frac{a}{2} .
$$


Then,

$$
\begin{aligned}
f_{M}(E(T)) & =N\left[\frac{\frac{a}{2}}{a}\right]^{N-1} \frac{1}{a}=\frac{N}{a}\left(\frac{1}{2}\right)^{N-1}, \\
& =\frac{N}{2} \frac{1}{E(T)}\left(\frac{1}{2}\right)^{N-1} .
\end{aligned}
$$

\subsubsection{Parabolic Distribution}

In this case, the probability density function of $T$ is defined in the following form:

$$
f_{T}(t)= \begin{cases}\frac{6}{a^{3}} t(a-t), & 0 \leq t \leq a \\ 0, & a<t\end{cases}
$$

where $a$ is some positive constant (Figure 1). Hence,

$$
F_{T}(t)=\left\{\begin{array}{lc}
\frac{3}{a^{2}} t^{2}-\frac{2}{a^{3}} t^{3}, & 0 \leq t \leq a \\
1, & a<t
\end{array} .\right.
$$

From these equations,

$$
f_{M}(t)=N\left[F_{T}(t)\right]^{N-1} f_{T}(t)=N\left[\frac{3}{a^{2}} t^{2}-\frac{2}{a^{3}} t^{3}\right]^{N-1} \frac{6}{a^{3}} t(a-t) .
$$

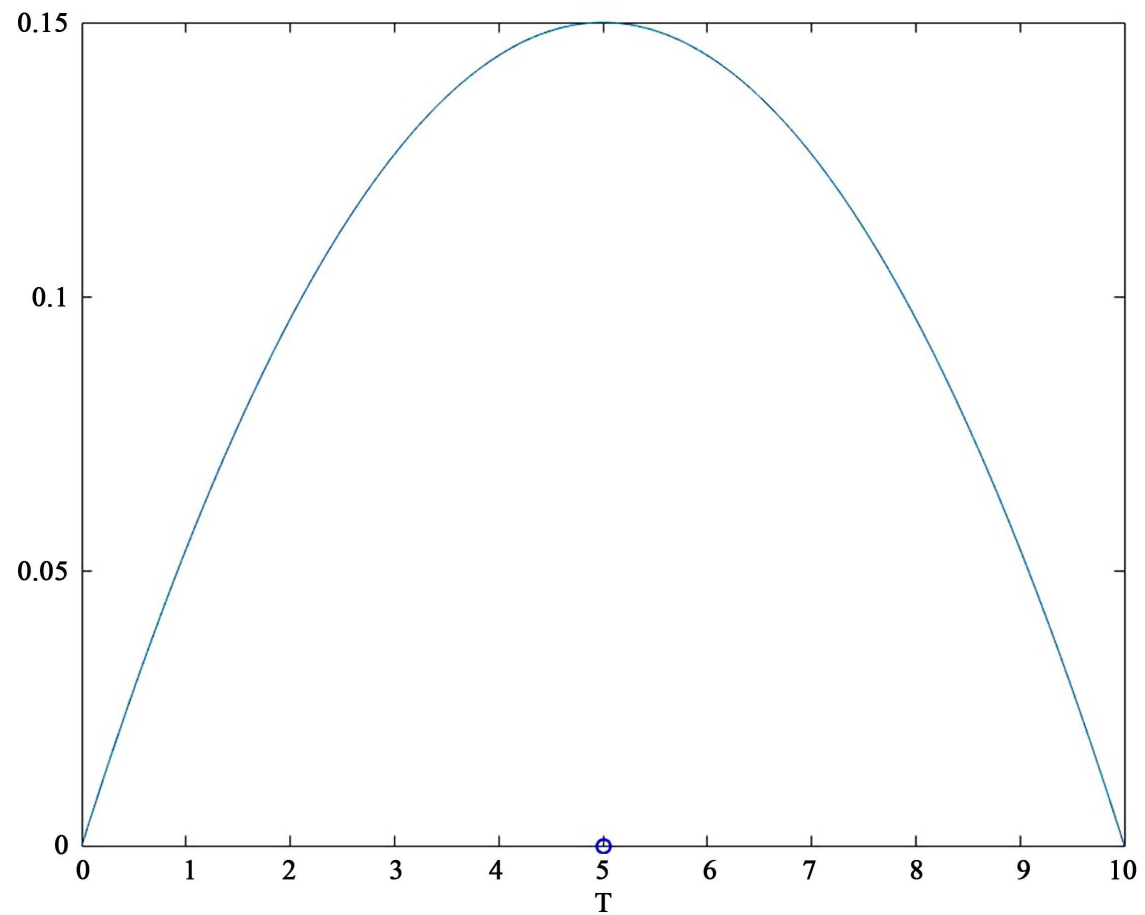

Figure 1. Parabolic distribution with $a=10$. The $\mathrm{x}$-axis is random variable $T$ and they-axis shows the probability density of $T$. Circle indicates the expected value of $T$. 
The expected value of $M$ is given by

$$
\begin{aligned}
E(M) & =\int_{0}^{a} t f_{M}(t) \mathrm{d} t=\int_{0}^{a} t N\left[\frac{3}{a^{2}} t^{2}-\frac{2}{a^{3}} t^{3}\right]^{N-1} \frac{6}{a^{3}} t(a-t) \mathrm{d} t \\
& =\left[t\left[\frac{3}{a^{2}} t^{2}-\frac{2}{a^{3}} t^{3}\right]^{N}\right]_{0}^{a}-\int_{0}^{a}\left[\frac{3}{a^{2}} t^{2}-\frac{2}{a^{3}} t^{3}\right]^{N} \mathrm{~d} t \\
& =a-a \sum_{k=0}^{N}\left(\begin{array}{l}
N \\
k
\end{array}\right) \frac{3^{k}(-2)^{N-k}}{3 N-k+1} .
\end{aligned}
$$

The expected value of the lifetime $T$ of each element is given as

$$
E(T)=\int_{0}^{a} t f_{T}(t) \mathrm{d} t=\frac{a}{2} .
$$

Then,

$$
\begin{gathered}
f_{M}(E(T))=N\left[\frac{3}{a^{2}}\left(\frac{a}{2}\right)^{2}-\frac{2}{a^{3}}\left(\frac{a}{2}\right)^{3}\right]^{N-1} \frac{6}{a^{3}} \frac{a}{2}\left(a-\frac{a}{2}\right)=\frac{3 N}{2 a}\left(\frac{1}{2}\right)^{N-1} \\
=\frac{3 N}{4} \frac{1}{E(T)}\left(\frac{1}{2}\right)^{N-1} .
\end{gathered}
$$

\subsubsection{Pointed Distribution Composed of Two Parabolas}

Here, the probability density function of random variable $T$, the lifetime of each element, is composed of two parabolas in the following form:

$$
f_{T}(t)=\left\{\begin{array}{lc}
\frac{3 t^{2}}{a^{2}(a+b)}, & 0 \leq t \leq a \\
\frac{3(t-(a+b))^{2}}{b^{2}(a+b)}, & a<t \leq a+b, \\
0, & a+b<t
\end{array}\right.
$$

where $a$ and $b$ are some positive constants (Figure 2). Hence,

$$
F_{T}(t)=\left\{\begin{array}{lc}
\frac{t^{3}}{a^{2}(a+b)}, & 0 \leq t \leq a \\
1+\frac{(t-(a+b))^{3}}{b^{2}(a+b)}, & a<t \leq a+b \\
1, & a+b<t
\end{array}\right.
$$

From these equations,

$$
\begin{aligned}
f_{M}(t) & =N\left[F_{T}(t)\right]^{N-1} f_{T}(t) \\
& = \begin{cases}N\left[\frac{t^{3}}{a^{2}(a+b)}\right]^{N-1} \frac{3 t^{2}}{a^{2}(a+b)}, & 0 \leq t \leq a \\
N\left[1+\frac{(t-(a+b))^{3}}{b^{2}(a+b)}\right]^{N-1} \frac{3(t-(a+b))^{2}}{b^{2}(a+b)}, & a<t \leq a+b\end{cases}
\end{aligned}
$$




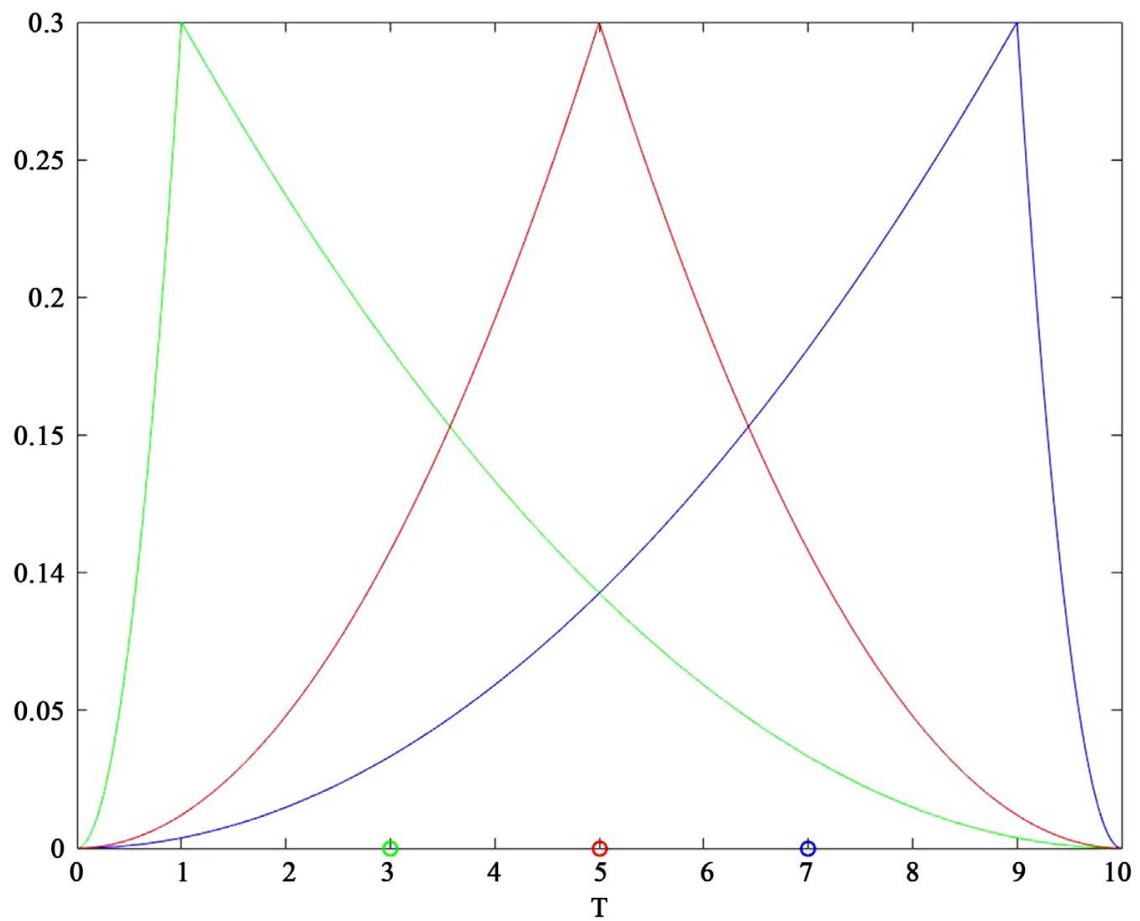

Figure 2. Three variations of a pointed distribution composed of two parabolas: $a=1, b=$ 9 (green); $a=5, b=5$ (red); and $a=9, b=1$ (blue). The $\mathrm{x}$-axis is random variable $T$ and the y-axis shows the probability density of $T$. Circles indicate the expected values of $T$.

The expected value of $M$ is given by

$$
\begin{aligned}
E(M)= & \int_{0}^{a+b} t f_{M}(t) \mathrm{d} t=\int_{0}^{a+b} t N\left[F_{T}(t)\right]^{N-1} f_{T}(t) \mathrm{d} t \\
= & \int_{0}^{a} t N\left[\frac{t^{3}}{a^{2}(a+b)}\right]^{N-1} \frac{3 t^{2}}{a^{2}(a+b)} \mathrm{d} t \\
& +\int_{a}^{a+b} t N\left[1+\frac{(t-(a+b))^{3}}{b^{2}(a+b)}\right]^{N-1} \frac{3(t-(a+b))^{2}}{b^{2}(a+b)} \mathrm{d} t \\
= & (a+b)-\frac{a^{N+1}}{(3 N+1)(a+b)^{N}} \\
& +\sum_{k=0}^{N}\left(\begin{array}{l}
N \\
k
\end{array}\right) \frac{(-b)^{3(N-k)+1}}{(3(N-k)+1) b^{2(N-k)}(a+b)^{N-k}}
\end{aligned}
$$

The expected value of the lifetime $T$ of each element is given as

$$
E(T)=\int_{0}^{a+b} t f_{T}(t) \mathrm{d} t=\int_{0}^{a} t \frac{3 t^{2}}{a^{2}(a+b)} \mathrm{d} t+\int_{a}^{a+b} t \frac{3(t-(a+b))^{2}}{b^{2}(a+b)} \mathrm{d} t=\frac{3 a+b}{4} .
$$

Using a parameter $k, 0<k, b$ can be denoted as $k a$. Then,

$$
E(T)=\frac{3 a+b}{4}=\frac{3+k}{4} a .
$$

If $E(T) \leq a, 0<k \leq 1$ and 


$$
\begin{aligned}
f_{M}(E(t)) & =N\left[\frac{\left(\frac{3+k}{4} a\right)^{3}}{a^{2}(a+k a)}\right]^{N-1} \frac{3\left(\frac{3+k}{4} a\right)^{2}}{a^{2}(a+k a)}=\frac{3 N}{\frac{3+k}{4} a}\left(\frac{\left(\frac{3+k}{4}\right)^{3}}{1+k}\right)^{N} \\
& \leq 3 N \frac{1}{E(T)}\left(\frac{1}{1+k}\right)^{N} .
\end{aligned}
$$

If $E(T)>a, 1<k$ and

$$
\begin{aligned}
f_{M}(E(t)) & =N\left[1+\frac{\left(\frac{3+k}{4} a-(a+k a)\right)^{3}}{(k a)^{2}(a+k a)}\right]^{N-1} \frac{3\left(\frac{3+k}{4} a-(a+k a)\right)^{2}}{(k a)^{2}(a+k a)} \\
& =N\left[\frac{37 k^{2}(1+k)-(3 k+1)}{64 k^{2}(1+k)}\right]^{N-1} \frac{3}{\frac{16}{\left(9+\frac{6}{k}+\frac{1}{k^{2}}\right)^{2}}} \\
& <N\left[\frac{37}{64}\right]^{N-1} \frac{3}{(1+k) a}=3 N \frac{1}{E(T)} \frac{\frac{3+k}{4}}{1+k}\left[\frac{37}{64}\right]^{N-1} \\
& <3 N \frac{1}{E(T)}\left[\frac{37}{64}\right]^{N-1}
\end{aligned}
$$

Then, if $k=1$, that is $a=b, E(T)=a$ and

$$
f_{M}(E(t))=N\left[\frac{a^{3}}{a^{2}(a+a)}\right]^{N-1} \frac{3 a^{2}}{a^{2}(a+a)}=\frac{3 N}{2} \frac{1}{E(T)}\left(\frac{1}{2}\right)^{N-1} .
$$

\section{Calculation Results}

\subsection{Exponential Distribution}

Two cases, $\lambda=0.1$ and 0.2 , are examined when the probability distribution is an exponential distribution. The parameter $\lambda$ can be selected arbitrarily. The value of $E(T)$ is the inverse of $\lambda: 10$ at $\lambda=0.1,5$ at $\lambda=0.2$. Hence, $\lambda$ is selected so that $E(T)$ of exponential distribution is comparable with $E(T)$ of the other distributions of finite range. Figure 3 shows that the critical number of elements for group survival are 22 at $\lambda=0.1$ and $\lambda=0.2$. In either case, the probability distribution of $M$ shifts to the right as $N$, the number of elements in the group, increases, and the value of $E(M)$ becomes more distant from $E(T)$. Because $0 \leq T<$ $\infty$, the value of $E(M)$ increases without limitation as $N$ increases. From Equation (14), $f_{M}(E(T))$ is inversely proportional to $E(T)$ and decreases as $N$ increases.

\subsection{Finite-Range Probability Distributions}

\subsubsection{Uniform Distribution}

A uniform distribution of a random variable, $T: 0 \leq T \leq 50$, is examined. Figure 4 shows that the critical number of elements needed for group survival are 10. In contrast to the case for exponential distribution, only the left half of the probability 

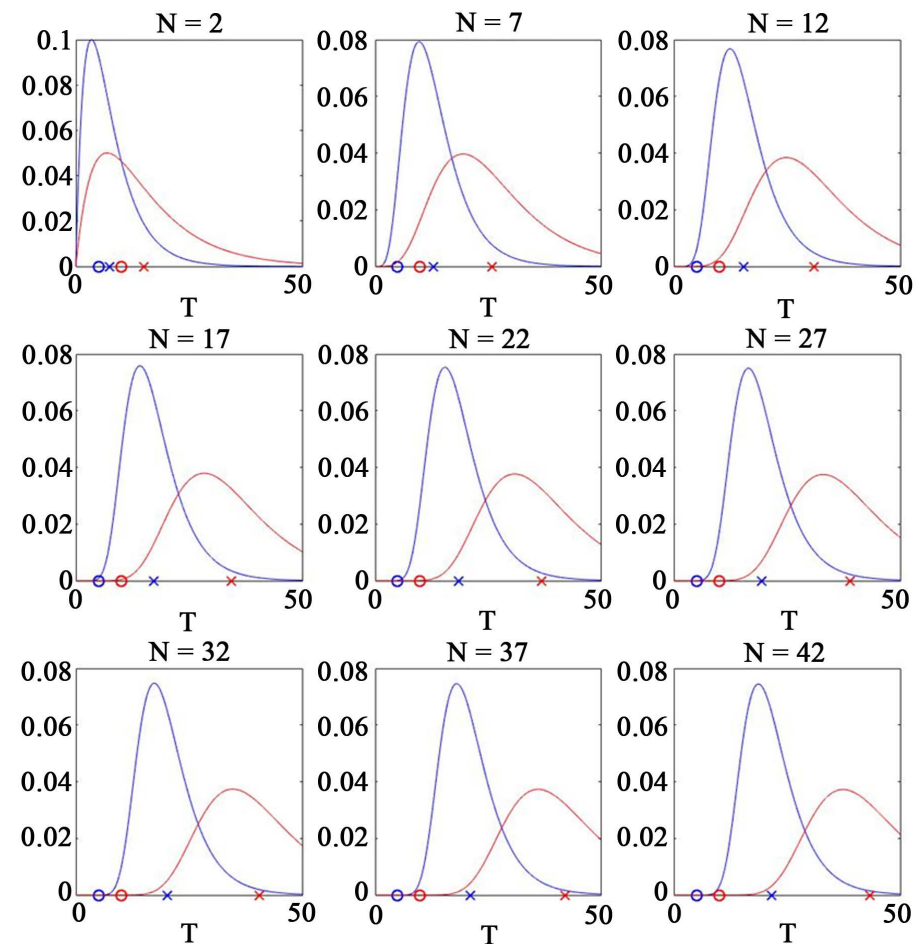

Figure 3. Two cases of the exponential distribution: $\lambda=0.1$ (red) and 0.2 (blue). The $\mathrm{x}$-axis is random variable $T$ and the y-axis shows the probability density of $T . N$ is the number of the elements. Circles indicate the expected values of $T$ and crosses indicate the expected value of the maximum lifetime of the elements.
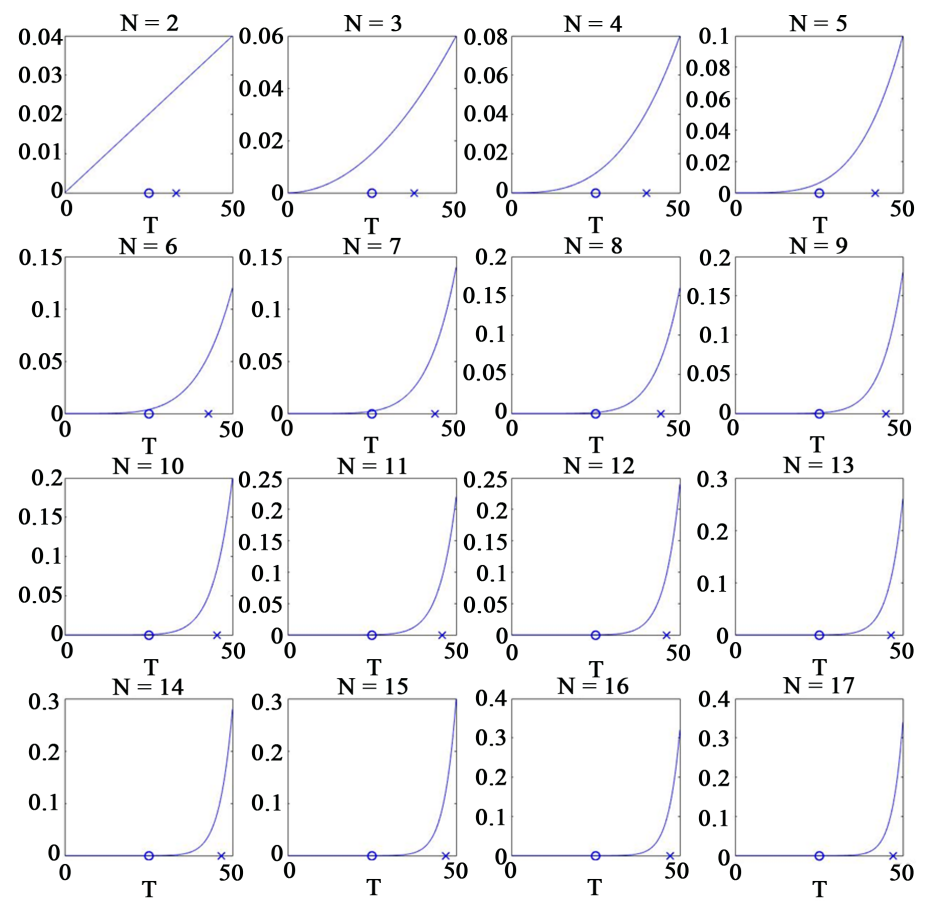

Figure 4. A uniform distribution of a random variable, $T: 0 \leq T \leq 50$. The $\mathrm{x}$-axis is a random variable, $T$ and the y-axis shows the probability density of $T$. $N$, number of the elements. Circles indicate the expected values of $T$ and crosses indicate the expected value of the maximum lifetime of the elements, respectively. 
distribution of $M$ shifts to the right as $N$ increases, and the value of $E(M)$ becomes more distant from $E(T)$ and approaches the upper limit of $T=50$. From Equation (21), $f_{M}(E(T))$ is inversely proportional to $E(T)$ and decreases as $N$ increases.

\subsubsection{Parabolic Distribution}

Two cases of $a=5$ and $a=10$ are examined for a parabolic distribution. The ranges of random variable $T$ are $0 \leq T \leq 5$ and $0 \leq T \leq 10$, respectively. Figure 5 shows that the critical number of elements needed for group survival are about 10 for both cases. Only the left half of the probability distribution of $M$ shifts to the right as $N$ increases, and the value of $E(M)$ becomes more distant from $E(T)$ and approaches the upper limit of $T$ as $N$ increases. From Equation (28), $f_{M}(E(T))$ is inversely proportional to $E(T)$ and decreases as $\mathrm{N}$ increases.

\subsubsection{Pointed Distribution Composed of Two Parabolas}

Three cases when the values of $(a, b)$ are $(1,9),(5,5)$ and $(9,1)$ are examined here. The ranges of random variable $T$ are equally $0 \leq T \leq 10$. Figure 6 shows that the critical numbers of elements needed for group survival are 12,11, and 9, respectively. Only the left half of the probability distribution of $M$ shifts to the right as $N$ increases, and the value of $E(M)$ becomes more distant from $E(T)$. In
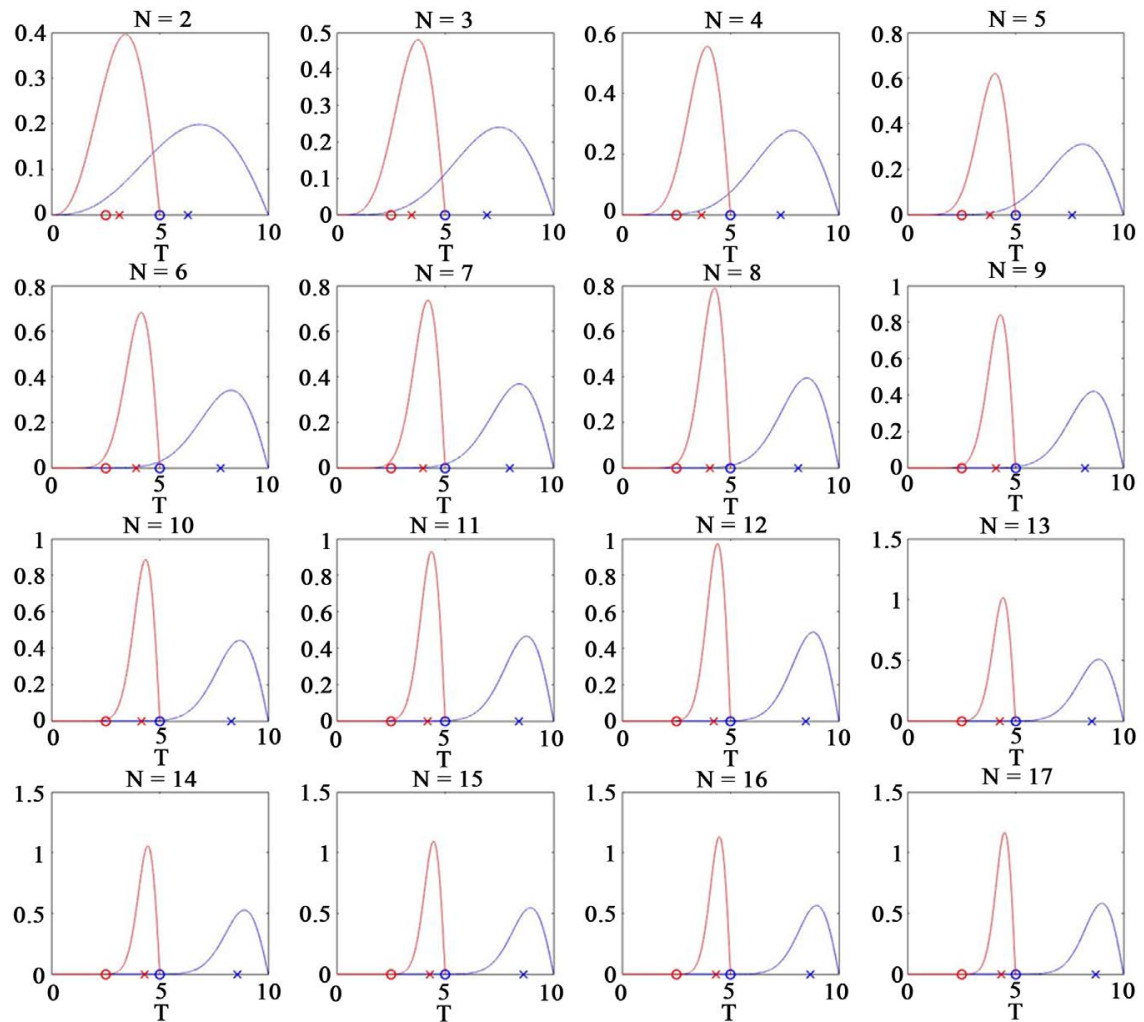

Figure 5. Two cases of parabolic distribution: $a=5$ (red) and $a=10$ (blue). The $\mathrm{x}$-axis is a random variable, $T$ and the $y$-axis shows the probability density of $T$. $N$, number of the elements. Circles indicate the expected values of $T$ and crosses indicate the expected value of the maximum lifetime of the elements. 

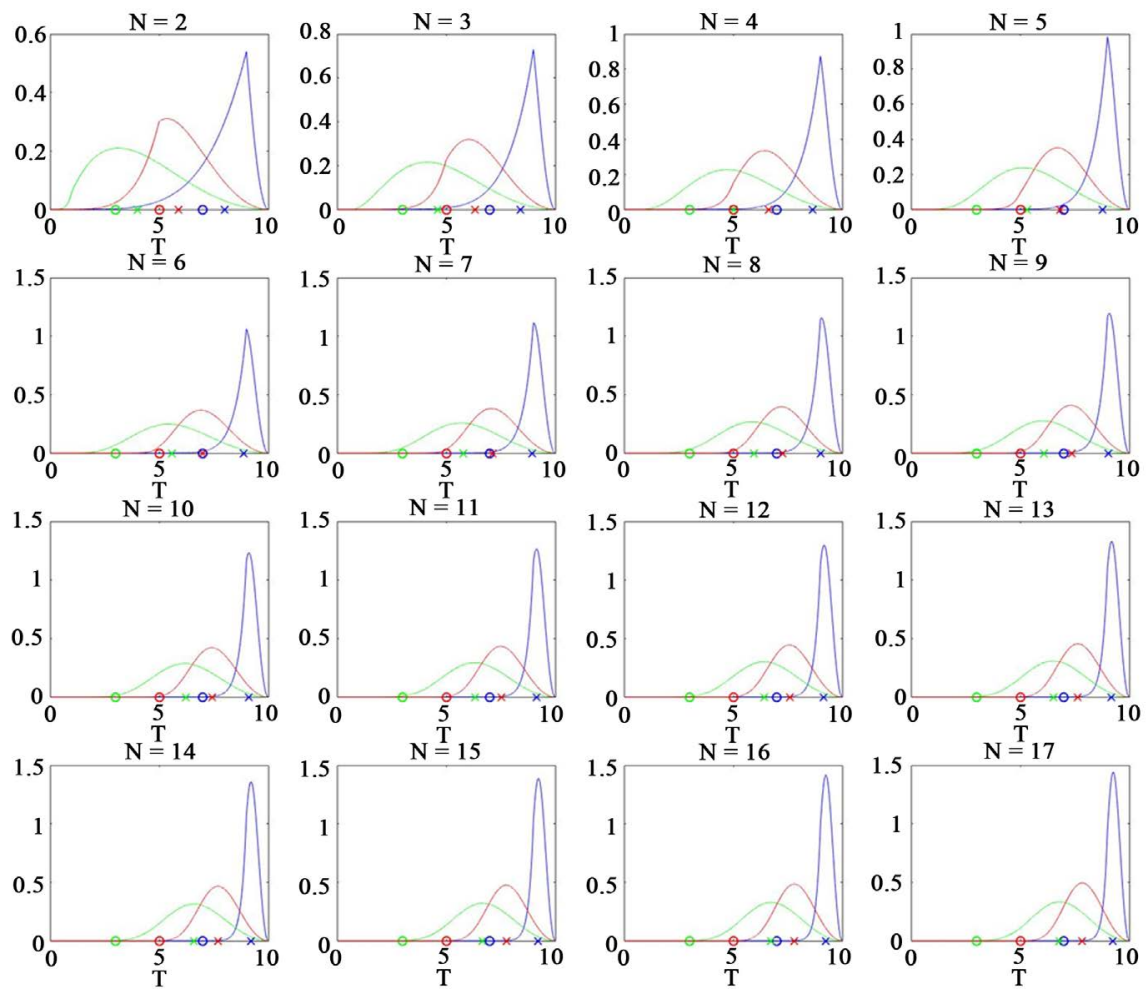

Figure 6. Three variations of pointed distribution composed of 2 parabolas: $a=1, b=9$ (green); $a=5, b=5$ (red); and $a=9, b=1$ (blue). The $\mathrm{x}$-axis is a random variable, $T$ and the y-axis shows the probability density of $T$. $N$, number of the elements. Circles indicate the expected values of $T$ and crosses indicate the expected value of the maximum lifetime of the elements.

either case, the value of $E(M)$ approaches the upper limit of $T$ as $N$ increases. From Equations (35) and (36), $f_{M}(E(T))$ is inversely proportional to $E(T)$ and decreases as $N$ increases. Particularly, $f_{M}(E(T))$ decreases as $\left(\frac{1}{2}\right)^{N-1}$ when $a=5$ and $b=5$.

\section{Discussion}

The findings of the calculations and their interpretations are as follows.

\subsection{Exponential Distribution}

In both cases of $\lambda=0.1$ and 0.2 , the expected value of the maximum lifetime of the group $E(M)$ increases as the number of elements $N$ increases. Because random variable $T$ has no limits, the value of $E(M)$ continues to increase. If the system of a group has a memoryless property with respect to lifetime, the lifetimes follow an exponential distribution. Although it may be difficult to determine such a system in the real world, cancer may be one possible example. A cancer cell appears suddenly and proliferates by cell division so that it increases to a mass of 1 billion cells over 10 - 20 years [14]. The lifetime of a cancer cell seems to be rather long, irrespective of aging and arteriosclerosis. Hence, the lifetimes 
in this system are assumed to have a memoryless property, which indicates that the lifetimes of cancer cells follow an exponential distribution. Because $E(T)$ is large and $f_{M}(E(T))$ is inversely proportional to $E(T)$ from Equation (14), the critical number of elements needed for group survival is rather small. Hence, if radiation therapy cannot destroy an instance of cancer completely, the cancer that survives it will grow back. From the perspective of probability, this is the reason that a large number of cancers are untreatable. Even if radiation therapy reduces the lifetime of cancer cells by half, i.e., $E(T)=10$ at $\lambda=0.1 \Rightarrow E(T)=5$ at $\lambda=0.2$, the mass will return if a few cells survive.

\subsection{Finite-Range Probability Distributions}

\subsubsection{Uniform Distribution}

It is difficult to find an example in the biological and medical fields that is likely to follow a uniform distribution. If the lifetimes of the elements in a group follow a uniform distribution, the diversity of the lifetimes is rather remarkable. Because the expected value of the maximum life time $E(M)$ is almost equal to the upper limit $a$ of the range of random variable $T: E(M)=\frac{N}{N+1} a$, the group will continue to work even under various disturbances. Because $f_{M}(E(T))$ is inversely proportional to $2^{N-1}$, from Equation (21), it is suggested that if the group has more than 10 elements, it will continue to function.

\subsubsection{Parabolic Distribution}

The parabolic distribution approximates a normal distribution with a finite range. Because the normal distribution appears universally in various fields due to the central limit theorem, the parabolic distribution may be applicable in the biological and medical fields. Two cases of $a=5$ and $a=10$ are examined, giving the ranges of a random variable $T$ as $0 \leq T \leq 5$ and $0 \leq T \leq 10$, respectively. If $a$ is reduced by half ( $a=10 \Rightarrow a=5$ in Figure 5 ), $E(M)$ is not much longer than $\mathrm{E}(T)$ when the number of elements in the group is less than 5 . This finding suggests that the group consisting of a few of elements is not robust against various disturbances. In contrast, when $a=10, E(M)$ is much longer than $E(T)$. In both cases, the critical number of elements needed for group survival is 10. From Equation $(28), f_{M}(E(T))$ is inversely proportional to $2^{N-1}$. These findings indicate that groups consisting of more than 10 elements may be robust against various disturbances. If the arterial flow to the visual cortex is suppressed by arteriosclerosis or infarction so that the lifetimes of neurons of the minicolumns are reduced by half, any subparts consisting of six pyramidal cells in the monkey primary visual cortex may not survive. However, because the functional column consists of hundreds of minicolumns, it is presumed that it can respond to similar visual patterns.

\subsubsection{Pointed Distribution Composed of Two Parabolas}

This distribution approximates a normal distribution with higher kurtosis. The ranges of random variable $T$ are $0 \leq T \leq 10$ in all three cases. When $a=1$ and $b=$ 
9, the distribution approximates positive skewness because $E(T)$ is in the left half of the range of $T$. When $a=5$ and $b=5$, the distribution approximates zero skewness because $E(T)$ is in the middle of the range of $T$. When $a=9$ and $b=1$, the distribution approximates negative skewness because $\mathrm{E}(T)$ is in the right half of the range of $T$. In all three cases, $E(M)$ shifts to the right. The critical number elements needed for group survival $(12 \Rightarrow 11 \Rightarrow 9)$ decreases as the skewness decreases (Figure 6). When $a=5$ and $b=5, E(M)$ at $N=17$ is smaller than $E(M)$ at $N=3$ when $a=9$ and $b=1$. Similarly, when $a=1$ and $b=9, E(M)$ at $N=17$ is smaller than $E(M)$ at $N=3$ when $a=9$ and $b=1$. These findings suggest that the group is even more robust against various disturbances when $a=9$ and $b=1$ than in other cases. When $a=b, f_{M}(E(T))$ is inversely proportional to $2^{N-1}$ from Equation (37). This indicates that a group consisting of more than 10 elements may be robust against various disturbances. The hippocampus and dentate gyrus play important roles in memory and learning. The axon of each granule cell in the dentate gyrus gives rise to approximately seven primary collaterals [15]. The hippocampal formation, which includes the hippocampus and dentate gyrus, is vulnerable to ischemia, in which memory and learning are damaged [16]. If the lifetimes follow a pointed distribution composed of two parabolas, seven primary collaterals may be insufficient for proper functionality because seven is less than the critical number of elements needed for group survival.

\subsection{Study Limitations}

Although there are many types of probability distributions, only four probability distributions were examined in this study. Because the exponential distribution is the probability distribution that describes the time between events in a process in which events occur independently at a constant average rate, it is a good choice for system modelling. However, the random variable has no upper limit in this distribution. Therefore, probability distributions with finite ranges were also examined. The uniform distribution is used as an example in which the random variable has the diversity with even probability. The parabolic distribution and pointed distribution composed of two parabolas are employed as approximations to a normal distribution.

\section{Conclusion}

This study examined how the lifetime of a group consisting of several elements depends on the number of elements and the probability distribution of their lifetimes. Four probability distributions were studied: an exponential distribution, a uniform distribution, a parabolic distribution, and a pointed distribution composed of two parabolas. The latter three distributions have a finite range. The probability distribution of the maximum life time $M$ shifts to the right as the number of elements $N$ increases, and the expected value $E(T)$ of the lifetime of each element eventually becomes less than its lower limit. Hence, if $N$ is larger than the critical number of elements needed for group survival, the lifetime of 
the group will be longer than $E(T)$. The critical number of elements needed for group survival is in inverse proportion to $E(T)$ regardless of the probability distributions used. For an exponential distribution, it decreases as $\left(\frac{2}{3}\right)^{N-1}$ and as $\left(\frac{1}{2}\right)^{N-1}$ for the other distributions. Because a probability distribution defined over a finite range is assumed to be reasonable in practice, a group consisting of more than 10 elements should survive well.

\section{Acknowledgements}

We thank Kim Moravec, $\mathrm{PhD}$, from Edanz Group

(http://www.edanzediting.com/ac) for editing a draft of this manuscript.

\section{Conflict of Interests}

The author declares that there are no conflicts of interest regarding the publication of this paper.

\section{References}

[1] Osaka, M. (2017) Modified Kuramoto Phase Model for Simulating Cardiac Pacemaker Cell Synchronization. Applied Mathematics, 8, 1227-1238. https://doi.org/10.4236/am.2017.89092

[2] Glass, L. (2001) Synchronization and Rhythmic Processes in Physiology. Nature, 410, 277-284. https://doi.org/10.1038/35065745

[3] Michaels, D.C., Matyas, E.P. and Jalife, J. (1986) Dynamic Interactions and Mutual Synchronization of Sinoatrial Node Pacemaker Cells. A Mathematical Model. Cir culation Research, 58, 706-720. https://doi.org/10.1161/01.RES.58.5.706

[4] Rodriguez, R.D. and Schocken, D.D. (1990) Update on Sick Sinus Syndrome, a Cardiac Disorder of Aging. Geriatrics, 45, 26-30, 33-36.

[5] Fotenos, A.F., Mintun, M.A., Snyder, A.Z., Morris, J.C. and Buckner, R.L. (2008) Brain Volume Decline in aging: Evidence for a Relation between Socioeconomic Status, Preclinical Alzheimer Disease, and Reserve. Archives of Neurology, 65, 113-120. https://doi.org/10.1001/archneurol.2007.27

[6] Mecocci, P., MacGarvey, U., Kaufman, A.E., Koontz, D., Shoffner, J.M., Wallace, D.C. and Beal, M.F. (1993) Oxidative Damage to Mitochondrial DNA Shows Marked Age-Dependent Increases in Human Brain. Annals of Neurology, 34, 609-616. https://doi.org/10.1002/ana.410340416

[7] Piguet, O., Ridley, L., Grayson, D.A., Bennett, H.P., Creasey, H., Lye, T.C. and Broe, G.A. (2003) Are MRI White Matter Lesions Clinically Significant in the 'Old-Old'? Evidence from the Sydney Older Persons Study. Dementia and Geriatric Cognitive Disorders, 15, 143-150. https://doi.org/10.1159/000068482

[8] Pakkenberg H. (1966) The Number of Nerve Cells in the Cerebral Cortex of Man. Journal of Comparative Neurology, 128, 17-20. https://doi.org/10.1002/cne.901280103

[9] Jones, E.G. (2000) Microcolumns in the Cerebral Cortex. Proceedings of the National Academy of Sciences U.S.A., 97, 5019-5021.

https://doi.org/10.1073/pnas.97.10.5019 
[10] Maruoka, H., Kubota, K., Kurokawa, R., Tsuruno, S. and Hosoya, T. (2011) Periodic Organization of a Major Subtype of Pyramidal Neurons in Neocortical Layer V. Journal of Neuroscience, 31, 18522-18542. https://doi.org/10.1523/JNEUROSCI.3117-11.2011

[11] Peters, A. and Sethares, C. (1996) Myelinated Axons and the Pyramidal Cell Modules in Monkey Primary Visual Cortex. Journal of Comparative Neurology, 365, 232-255. https://doi.org/10.1002/(SICI)1096-9861(19960205)365:2<232::AID-CNE3>3.0.CO; $\underline{2-6}$

[12] Fujita, I., Tanaka, K., Ito, M. and Cheng, K. (1992) Columns for Visual Features of Objects in Monkey Inferotemporal Cortex. Nature, 360, 343-346. https://doi.org/10.1038/360343a0

[13] Nahin, P.J. (2014) Will You Be Alive 10 Years from Now? Princeton University Press, Princeton and Oxford. https://doi.org/10.1515/9781400848379

[14] Tubiana, M. (1989) Tumor Cell Proliferation Kinetics and Tumor Growth Rate. Acta Oncologica, 28, 113-121. https://doi.org/10.3109/02841868909111193

[15] Claiborne, B.J., Amaral, D.G. and Cowan, W.M. (1986) A Light and Electron Microscopic Analysis of the Mossy Fibers of the Rat Dentate Gyrus. Journal of Comparative Neurology, 246, 435-458. https://doi.org/10.1002/cne.902460403

[16] Nikonenko, A.G., Radenovic, L., Andjus, P.R. and Skibo, G.G. (2009) Structural Features of Ischemic Damage in the Hippocampus. Anatomical Record, 292, 1914-1921. https://doi.org/10.1002/ar.20969 